\title{
A NOTE ON THE PAPER "NECESSARY AND SUFFICIENT OPTIMALITY CONDITIONS USING CONVEXIFACTORS FOR MATHEMATICAL PROGRAMS WITH EQUILIBRIUM CONSTRAINTS"
}

\author{
Nazih AbDerrazzaK Gadhi*
}

\begin{abstract}
In this work, some counterexamples are given to refute some results in the paper by Kohli [RAIRO:OR 53 (2019) 1617-1632]. We correct the fault in some of his results.
\end{abstract}

Mathematics Subject Classification. 90C30, 90C46, 49J52.

Received June 24, 2020. Accepted September 18, 2021.

Comment on: RAIRO-Oper. Res. 53 (2019) 1617-1632. https://doi.org/10.1051/ro/2018084

\section{INTRODUCTION}

Mathematical programs with equilibrium constraints have been investigated by many authors. In the paper [8], the author investigated the following mathematical programs with equilibrium constraints

$$
(\mathrm{MPEC}):\left\{\begin{array}{l}
\text { Minimize } f(x) \\
\text { s.t. }\left\{\begin{array}{l}
g(x) \leq 0, h(x)=0, \\
G(x) \geq 0, H(x) \geq 0, G(x)^{\top} H(x)=0,
\end{array}\right.
\end{array}\right.
$$

where $f: \mathbb{R}^{n} \rightarrow \mathbb{R}, g: \mathbb{R}^{n} \rightarrow \mathbb{R}^{m}, h: \mathbb{R}^{n} \rightarrow \mathbb{R}^{p}, G: \mathbb{R}^{n} \rightarrow \mathbb{R}^{l}$ and $H: \mathbb{R}^{n} \rightarrow \mathbb{R}^{l}$. Under a nonsmooth constraint qualification $\left(\partial^{*}-G C Q\right)$ given in terms of convexifactors, the author established first order necessary optimality condition for (MPEC). The main theorem, where the author gave necessary optimality conditions, is Theorem 4.4 of $[8]$.

In this article, we show that necessary optimality conditions given by Kohli [8] are not correct. In support of our remarks, some counterexamples are given (see Example 3.1 and Rem. 3.3) and some reasoning mistakes in the proof of the main result ([8], Thm. 4.4) are highlighted (see Rems. 3.2, 3.3 and 4.2). Finally, we present corrected versions of his results. Theorem 4.5 is actually a corrected version of Theorem 4.4 in [8].

The rest of the paper is organized in this way: Section 2 contains basic definitions and preliminary material. Counterexamples and comments are given in Section 3. Section 4 addresses our main results (corrected optimality conditions). A conclusion is given in Section 5 .

Keywords. Convexifactor, constraint qualifications, mathematical programs with equilibrium constraints, optimality conditions. LAMA, USMBA, FSDM, Department of Mathematics, Fes, Morocco.

*Corresponding author: ngadhi@hotmail.com.

(C) The authors. Published by EDP Sciences, ROADEF, SMAI 2021 


\section{Preliminaries}

Throughout this section, let $\mathbb{R}^{n}$ be the usual $n$-dimensional Euclidean space. Given a nonempty subset $S$ of $\mathbb{R}^{n}$, the closure, convex hull, and convex cone (including the origin) generated by $S$ are denoted respectively by $c l S$, conv $S$ and pos $S$. The negative polar cone of $S$ is defined by

$$
S^{-}:=\left\{v \in \mathbb{R}^{n} \mid\langle x, v\rangle \leq 0, \forall x \in S\right\} .
$$

The contingent cone $T(S, x)$ to $S$ at $x \in c l S$ is defined by

$$
T(S, x)=\left\{v \in \mathbb{R}^{n}: \exists t_{n} \downarrow 0 \text { and } \exists v_{n} \rightarrow v \text { such that } x+t_{n} v_{n} \in S, \forall n \in \mathbb{N}\right\} .
$$

Let $f: \mathbb{R}^{n} \rightarrow \mathbb{R} \cup\{+\infty\}$ be a given function and let $x \in \mathbb{R}^{n}$ where $f(x)$ is finite. The expressions

$$
f_{d}^{-}(x, v)=\liminf _{t \backslash 0}[f(x+t v)-f(x)] / t \text { and } f_{d}^{+}(x, v)=\limsup _{t \searrow 0}[f(x+t v)-f(x)] / t
$$

signify, respectively, the lower and upper Dini directional derivatives of $f$ at $x$ in the direction $v$.

Definition 2.1 ([2]). The function $f: \mathbb{R}^{n} \rightarrow \mathbb{R} \cup\{+\infty\}$ is said to have an upper convexifactor $\partial^{u} f(x)$ at $x$ if $\partial^{u} f(x) \subseteq \mathbb{R}^{n}$ is closed and, for each $v \in \mathbb{R}^{n}$,

$$
f_{d}^{-}(x, v) \leq \sup _{x^{*} \in \partial^{u} f(x)}\left\langle x^{*}, v\right\rangle .
$$

The function $f: \mathbb{R}^{n} \rightarrow \mathbb{R} \cup\{+\infty\}$ is said to have an upper semiregular convexifactor $\partial^{u s} f(x)$ at $x$ if $\partial^{u s} f(x)$ is an upper convexifactor at $x$ and, for each $v \in \mathbb{R}^{n}$,

$$
f_{d}^{+}(x, v) \leq \sup _{x^{*} \in \partial^{u s} f(x)}\left\langle x^{*}, v\right\rangle .
$$

\section{Counterexamples And COMments}

The following example shows that Theorem 4.4 of [8] is not correct.

Example 3.1. Consider the optimization problem (MPEC) where

$$
\begin{aligned}
& f\left(x_{1}, x_{2}, x_{3}\right):=x_{1}+x_{2}-2 x_{3}, g\left(x_{1}, x_{2}, x_{3}\right):=x_{3}, \\
& h\left(x_{1}, x_{2}, x_{3}\right):=0, G_{1}\left(x_{1}, x_{2}, x_{3}\right):=x_{1}, G_{2}\left(x_{1}, x_{2}, x_{3}\right):=x_{2}, H_{1}\left(x_{1}, x_{2}, x_{3}\right):=x_{2} \text { and } H_{2}\left(x_{1}, x_{2}, x_{3}\right):=x_{1} .
\end{aligned}
$$

On the one hand, the origin is the unique minimizer of (MPEC). On the other hand, it can be seen that $\partial^{u s} f(\bar{x}):=\{(1,1,-2)\}$ is a bounded upper semiregular convexifactor of $f$ at $\bar{x}:=(0,0,0)$. Moreover,

$$
\begin{aligned}
\partial^{u} g(\bar{x}) & :=\{(0,0,1)\}, \partial^{u} h(\bar{x}):=\{(0,0,0)\} \\
\partial^{u} G_{1}(\bar{x}) & :=\{(1,0,0)\}, \partial^{u}\left(-G_{1}\right)(\bar{x}):=\{(-1,0,0)\}, \partial^{u} G_{2}(\bar{x}):=\{(0,1,0)\}, \partial^{u}\left(-G_{2}\right)(\bar{x}):=\{(0,-1,0)\}, \\
\partial^{u} H_{1}(\bar{x}) & :=\{(0,1,0)\}, \partial^{u}\left(-H_{1}\right)(\bar{x}):=\{(0,-1,0)\}, \partial^{u} H_{2}(\bar{x}):=\{(1,0,0)\} \text { and } \partial^{u}\left(-H_{2}\right)(\bar{x}):=\{(-1,0,0)\}
\end{aligned}
$$

are upper convexifactors of $g, h, G_{1},-G_{1}, G_{2},-G_{2}, H_{1},-H_{1}, H_{2}$ and $-H_{2}$ at $\bar{x}$ respectively. Remark that $B=\{1,2\}$.

- The feasible set $K$ of $(\mathrm{MPEC})$ is $K=\left(\{0\} \times \mathbb{R}^{+} \times \mathbb{R}^{-}\right) \cup\left(\mathbb{R}^{+} \times\{0\} \times \mathbb{R}^{-}\right)$. Consequently,

$$
T(K, \bar{x})=\left(\{0\} \times \mathbb{R}^{+} \times \mathbb{R}^{-}\right) \cup\left(\mathbb{R}^{+} \times\{0\} \times \mathbb{R}^{-}\right) \text {and clconv } T(K, \bar{x})=\mathbb{R}^{+} \times \mathbb{R}^{+} \times \mathbb{R}^{-} .
$$

- $\partial^{*}-G C Q\left(B_{1}, B_{2}\right)$ holds for all $\left(B_{1}, B_{2}\right) \in P(B)$ at $\bar{x}$. 
- If $B_{1}=\{1\}$ and $B_{2}=\{2\}$, then $\partial^{*}-G C Q\left(B_{1}, B_{2}\right)$ holds at $\bar{x}$. Indeed,

$$
\left(\begin{array}{l}
\left(\operatorname{conv} \partial^{u} g(\bar{x})\right) \bigcup\left(\operatorname{conv} \partial^{u} h(\bar{x})\right) \bigcup\left(\operatorname{conv} \partial^{u} G_{2}(\bar{x}) \cup \operatorname{conv} \partial^{u}\left(-G_{2}\right)(\bar{x})\right) \\
\bigcup\left(\operatorname{conv} \partial^{u} H_{1}(\bar{x}) \cup \operatorname{conv} \partial^{u}\left(-H_{1}\right)(\bar{x})\right) \bigcup \operatorname{conv} \partial^{u}\left(-G_{1}\right)(\bar{x}) \bigcup\left(\operatorname{conv} \partial^{u}\left(-H_{2}\right)(\bar{x})\right)
\end{array}\right)^{-}=\mathbb{R}^{+} \times\{0\} \times \mathbb{R}^{-} .
$$

- If $B_{1}=\{2\}$ and $B_{2}=\{1\}$, then $\partial^{*}-G C Q\left(B_{1}, B_{2}\right)$ holds at $\bar{x}$. Indeed,

$$
\left(\begin{array}{l}
\left(\operatorname{conv} \partial^{u} g(\bar{x})\right) \bigcup\left(\operatorname{conv} \partial^{u} h(\bar{x})\right) \bigcup\left(\operatorname{conv} \partial^{u} G_{1}(\bar{x}) \cup \operatorname{conv} \partial^{u}\left(-G_{1}\right)(\bar{x})\right) \\
\bigcup\left(\operatorname{conv} \partial^{u} H_{2}(\bar{x}) \cup \operatorname{conv} \partial^{u}\left(-H_{2}\right)(\bar{x})\right) \bigcup \operatorname{conv} \partial^{u}\left(-G_{2}\right)(\bar{x}) \bigcup\left(\operatorname{conv} \partial^{u}\left(-H_{1}\right)(\bar{x})\right)
\end{array}\right)^{-}=\{0\} \times \mathbb{R}^{+} \times \mathbb{R}^{-} .
$$

- If $B_{1}=\emptyset$ and $B_{2}=\{1,2\}$, then $\partial^{*}-G C Q\left(B_{1}, B_{2}\right)$ holds at $\bar{x}$. Indeed,

$$
\left(\begin{array}{l}
\left(\operatorname{conv} \partial^{u} g(\bar{x})\right) \bigcup\left(\operatorname{conv} \partial^{u} h(\bar{x})\right) \bigcup\left(\operatorname{conv} \partial^{u} G_{1}(\bar{x}) \cup \operatorname{conv} \partial^{u}\left(-G_{1}\right)(\bar{x})\right) \\
\bigcup\left(\operatorname{conv} \partial^{u} G_{2}(\bar{x}) \cup \operatorname{conv} \partial^{u}\left(-G_{2}\right)(\bar{x})\right) \cup \operatorname{conv} \partial^{u}\left(-H_{1}\right)(\bar{x}) \bigcup\left(\operatorname{conv} \partial^{u}\left(-H_{2}\right)(\bar{x})\right)
\end{array}\right)^{-}=\{0\} \times\{0\} \times \mathbb{R}^{-} .
$$

- If $B_{1}=\{1,2\}$ and $B_{2}=\emptyset$, then $\partial^{*}-G C Q\left(B_{1}, B_{2}\right)$ holds at $\bar{x}$. Indeed,

$$
\left(\begin{array}{l}
\left(\operatorname{conv} \partial^{u} g(\bar{x})\right) \bigcup\left(\operatorname{conv} \partial^{u} h(\bar{x})\right) \bigcup\left(\operatorname{conv} \partial^{u} H_{1}(\bar{x}) \cup \operatorname{conv} \partial^{u}\left(-H_{1}\right)(\bar{x})\right) \\
\bigcup\left(\operatorname{conv} \partial^{u} H_{2}(\bar{x}) \cup \operatorname{conv} \partial^{u}\left(-H_{2}\right)(\bar{x})\right) \bigcup \operatorname{conv} \partial^{u}\left(-G_{1}\right)(\bar{x}) \bigcup\left(\operatorname{conv} \partial^{u}\left(-G_{2}\right)(\bar{x})\right)
\end{array}\right)^{-}=\{0\} \times\{0\} \times \mathbb{R}^{-} .
$$

- $\partial^{*}-G C Q$ holds at $\bar{x}$. Indeed,

$$
\left(\begin{array}{l}
\left(\operatorname{conv} \partial^{u} g(\bar{x})\right) \cup\left(\operatorname{conv} \partial^{u} h(\bar{x})\right) \\
\cup\left(\operatorname{conv} \partial^{u}\left(-G_{1}\right)(\bar{x}) \cup \operatorname{conv} \partial^{u}\left(-G_{2}\right)(\bar{x})\right) \\
\bigcup\left(\operatorname{conv} \partial^{u}\left(-H_{1}\right)(\bar{x}) \cup \operatorname{conv} \partial^{u}\left(-H_{2}\right)(\bar{x})\right)
\end{array}\right)^{-}=\mathbb{R}^{+} \times \mathbb{R}^{+} \times \mathbb{R}^{-} \subseteq \operatorname{clconv} T(K, \bar{x}) .
$$

- Observe that all hypotheses of Theorem 4.4 in [8] are satisfied, but $\bar{x}$ is not a $\partial^{*}$-strong stationary point as defined by Kohli ([8], Def. 4.1). Indeed, if there exists a vector $0 \neq\left(\lambda^{g}, \lambda^{h}, \lambda^{G}, \lambda^{H}, \mu^{G}, \mu^{H}\right) \in \mathbb{R} \times \mathbb{R} \times \mathbb{R}^{2} \times$ $\mathbb{R}^{2} \times \mathbb{R}^{2} \times \mathbb{R}^{2}$ such that

$$
\begin{aligned}
\lambda^{g}, \lambda^{h}, \lambda_{1}^{G}, \lambda_{2}^{G}, \lambda_{1}^{H}, \lambda_{2}^{H}, \mu_{1}^{G}, \mu_{2}^{G}, \mu_{1}^{H}, \mu_{2}^{H} \geq 0, \\
\lambda^{g}+\lambda^{h}+\lambda_{1}^{G}+\lambda_{2}^{G}+\lambda_{1}^{H}+\lambda_{2}^{H}+\mu_{1}^{G}+\mu_{2}^{G}+\mu_{1}^{H}+\mu_{2}^{H}=1
\end{aligned}
$$

and

$$
0 \in \operatorname{cl}\left[\begin{array}{l}
\operatorname{conv} \partial^{u s} f(\bar{x})+\lambda^{g} \operatorname{conv} \partial^{u} g(\bar{x})+\lambda^{h} \operatorname{conv} \partial^{u} h(\bar{x})+\lambda_{1}^{G} \operatorname{conv} \partial^{u}\left(-G_{1}\right)(\bar{x}) \\
+\lambda_{2}^{G} \operatorname{conv} \partial^{u}\left(-G_{2}\right)(\bar{x})+\lambda_{1}^{H} \operatorname{conv} \partial^{u}\left(-H_{1}\right)(\bar{x})+\lambda_{2}^{H} \operatorname{conv} \partial^{u}\left(-H_{2}\right)(\bar{x}) \\
+\mu_{1}^{G} \operatorname{conv} \partial^{u} G_{1}(\bar{x})+\mu_{1}^{H} \operatorname{conv} \partial^{u} H_{1}(\bar{x})+\mu_{2}^{G} \operatorname{conv} \partial^{u} G_{2}(\bar{x})+\mu_{2}^{H} \operatorname{conv} \partial^{u} H_{2}(\bar{x})
\end{array}\right]
$$

we get

$$
\left(\begin{array}{l}
0 \\
0 \\
0
\end{array}\right) \in \operatorname{cl}\left\{\left(\begin{array}{l}
1-\lambda_{1}^{G}-\lambda_{2}^{H}+\mu_{1}^{G}+\mu_{2}^{H} \\
1-\lambda_{2}^{G}-\lambda_{1}^{H}+\mu_{1}^{H}+\mu_{2}^{G} \\
-2+\lambda^{g}
\end{array}\right)\right\}
$$

Then,

$$
\left\{\begin{array}{l}
0=1-\lambda_{1}^{G}-\lambda_{2}^{H}+\mu_{1}^{G}+\mu_{2}^{H} \\
0=1-\lambda_{2}^{G}-\lambda_{1}^{H}+\mu_{1}^{H}+\mu_{2}^{G} \\
0=-2+\lambda^{g}
\end{array}\right.
$$

We have $\lambda^{g}=2$ while $\lambda^{g} \leq 1$ due to (3.1) and (3.2). A contradiction. 
Remark 3.2. Contrary to what is stated on page 1625 (line -1), it is impossible to deduce

$$
\lim _{k \rightarrow \infty}\left[\sum_{i \in I(\bar{x})} \lambda_{i k}^{g}+\sum_{i=1}^{p} \lambda_{i k}^{h}+\sum_{i \in A \cup B_{1} \cup B_{2}} \lambda_{i k}^{G}+\sum_{i \in D \cup B_{1} \cup B_{2}} \lambda_{i k}^{H}+\sum_{i \in A \cup B_{2}} \mu_{i k}^{G}+\sum_{i \in D \cup B_{1}} \mu_{i k}^{H}\right]:=1 .
$$

The author did not pay attention to the cone that precedes the convex hull in the previous formula (see line- 6 on page 1625). This error has seriously impacted the remaining of the proof of Theorem 4.4 from [8]. Since (3.3) is an essential part of the definition of the $\partial^{*}$-strong stationarity property, Theorem 4.4 of $[8]$ is also not correct. Notice that the boundedness of the sequence of the multipliers is neither acquired nor insured.

Remark 3.3. The main result ([8], Thm. 4.4), is based on Lemma 2.3 of [8]. However, this latter ([8], Lem. 2.3) is clearly incorrect, as setting

$$
A:=\left\{(x, y) \in \mathbb{R}^{2}: x<0, y<0\right\} \cup\{(0,0)\} \text { and } B:=\{(1,0)\}
$$

yields a simple counterexample. Unfortunately, this error impacted ([8], Thm. 4.4) and forced the author to add useless and cumbersome closures and convex hulls.

The following result is a corrected version of Lemma 2.3 from [8]. Being standard, the proof has been omitted.

Lemma 3.4. Let $\mathcal{B}$ a nonempty, convex and compact set and $\mathcal{A}$ be a convex cone. If

$$
\sup _{v \in \mathcal{B}}\langle v, d\rangle \geq 0, \quad \text { for all } d \in \mathcal{A}^{-}
$$

then $0 \in \mathcal{B}+\operatorname{cl} \mathcal{A}$.

\section{Optimality CONDitions}

In the following definition, we recall the generalized alternatively stationarity concept given by Ardali et al. ([1], Def. 4.3).

Definition 4.1 ([1]). A feasible point $\bar{x}$ of MPEC is said to be a generalized alternatively stationary point if there exists a vector $\left(\lambda^{g}, \lambda^{h}, \mu^{h}, \lambda^{G}, \lambda^{H}, \mu^{G}, \mu^{H}\right) \in \mathbb{R}^{m} \times \mathbb{R}^{2 p} \times \mathbb{R}^{2 l} \times \mathbb{R}^{2 l}$ such that

$$
0 \in\left[\begin{array}{l}
\operatorname{conv} \partial^{u s} f(\bar{x})+\sum_{i=1}^{m} \lambda_{i}^{g} \operatorname{conv} \partial^{u} g_{i}(\bar{x})+\sum_{i \in I^{\prime}} \mu_{i}^{h} \operatorname{conv} \partial^{u} h_{i}(\bar{x})+\sum_{i \in I^{\prime}} \lambda_{i}^{h} \operatorname{conv} \partial^{u}\left(-h_{i}\right)(\bar{x})+\sum_{i=1}^{l} \lambda_{i}^{G} \\
\times \operatorname{conv} \partial^{u}\left(-G_{i}\right)(\bar{x})+\sum_{i=1}^{l} \lambda_{i}^{H} \operatorname{conv} \partial^{u}\left(-H_{i}\right)(\bar{x})+\sum_{i=1}^{l} \mu_{i}^{G} \operatorname{conv} \partial^{u} G_{i}(\bar{x})+\sum_{i=1}^{l} \mu_{i}^{H} \operatorname{conv} \partial^{u} H_{i}(\bar{x})
\end{array}\right]
$$

with

$$
\lambda_{i}^{g} g_{i}(\bar{x})=0, \forall i \in I
$$

and

$$
\left\{\begin{array}{l}
\mu_{i}^{G}=0 \text { or } \mu_{i}^{H}=0, \forall i \in B, \\
\lambda_{i}^{G}=0, \mu_{i}^{G}=0, \forall i \in D, \\
\lambda_{i}^{H}=0, \mu_{i}^{H}=0, \forall i \in A, \\
\lambda_{i}^{G}, \lambda_{i}^{H}, \mu_{i}^{G}, \mu_{i}^{H} \geq 0, \forall i \in\{1, \ldots, l\}, \\
\lambda_{i}^{g} \geq 0, \forall i \in I=\{1, \ldots, m\}, \text { and } \lambda_{i}^{h} \geq 0, \mu_{i}^{h} \geq 0, \forall i \in I^{\prime}=\{1, \ldots, p\} .
\end{array}\right.
$$

Here,

$$
\begin{aligned}
& A:=\left\{i \in\{1, \ldots, l\}: G_{i}(\bar{x})=0, H_{i}(\bar{x})>0\right\} \\
& B:=\left\{i \in\{1, \ldots, l\}: G_{i}(\bar{x})=0, H_{i}(\bar{x})=0\right\} \\
& D:=\left\{i \in\{1, \ldots, l\}: G_{i}(\bar{x})>0, H_{i}(\bar{x})=0\right\}
\end{aligned}
$$


Remark 4.2. Contrary to Definition 10 of [8],

$$
\sum_{i=1}^{m} \lambda_{i}^{g}+\sum_{i=1}^{p} \lambda_{i}^{h}+\sum_{i=1}^{l} \lambda_{i}^{G}+\sum_{i=1}^{l} \lambda_{i}^{H}+\sum_{i=1}^{l} \mu_{i}^{G}+\sum_{i=1}^{l} \mu_{i}^{H}=1
$$

is not an integral part of Definition 4.1. It is this equality that distorted Kohli's result. Notice that Remark 4.2 of $[8]$ is not correct since condition for the sum of multipliers does not exist in $[4,6,10]$.

Remark 4.3. Notice that if all the functions are differentiable and the upper convexifactor is replaced by the upper regular convexifactor in the above stationary notion, then this notion reduces to the A-stationary condition given by Flegel and Kanzow [6] and by Flegel [3].

We shall need the following nonsmooth constraint qualification.

Definition 4.4. Let $\bar{x} \in K$ and $\left(B_{1}, B_{2}\right)$ be a partition of $B \neq \emptyset$. Suppose that $g_{i}, i \in I, h_{i},-h_{i}, i \in J$, $-G_{i}, G_{i}, i \in A \cup B,-H_{i}, H_{i}, i \in D \cup B$, admit upper convexifactors $\partial^{u} g_{i}(\bar{x}), i \in I, \partial^{u} h_{i}(\bar{x}), \partial^{u}\left(-h_{i}\right)(\bar{x})$, $i \in J, \partial^{u}\left(-G_{i}\right)(\bar{x}), \partial^{u} G_{i}(\bar{x}), i \in A \cup B, \partial^{u}\left(-H_{i}\right)(\bar{x}), \partial^{u} H_{i}(\bar{x}), i \in D \cup B$, respectively at $\bar{x}$. We say that $\partial^{*}-A C Q\left(B_{1}, B_{2}\right)$ holds at $\bar{x}$ if

$$
\mathcal{A}^{-} \subseteq \operatorname{clconv}(T(K, \bar{x}))
$$

where $K$ is the feasible set of (MPEC) and

$$
\begin{aligned}
\mathcal{A} & :=\left(\bigcup_{i \in I(\bar{x})} \operatorname{conv} \partial^{u} g_{i}(\bar{x})\right) \cup\left(\bigcup_{i \in I^{\prime}} \operatorname{conv} \partial^{u} h_{i}(\bar{x})\right) \cup\left(\bigcup_{i \in I^{\prime}} \operatorname{conv} \partial^{u}\left(-h_{i}\right)(\bar{x})\right) \\
& \cup\left(\bigcup_{i \in A \cup B_{2}}\left(\operatorname{conv} \partial^{u} G_{i}(\bar{x}) \cup \operatorname{conv} \partial^{u}\left(-G_{i}\right)(\bar{x})\right)\right) \cup\left(\bigcup_{i \in D \cup B_{1}}\left(\operatorname{conv} \partial^{u} H_{i}(\bar{x}) \cup \operatorname{conv} \partial^{u}\left(-H_{i}\right)(\bar{x})\right)\right) \\
& \cup\left(\bigcup_{i \in B_{1}} \operatorname{conv} \partial^{u}\left(-G_{i}\right)(\bar{x})\right) \cup\left(\bigcup_{i \in B_{2}} \operatorname{conv} \partial^{u}\left(-H_{i}\right)(\bar{x})\right) .
\end{aligned}
$$

The following result is the corrected version of Theorem 4.4 from [8].

Theorem 4.5. Let $\bar{x}$ be a local optimal solution of MPEC. Assume that $f$ is locally Lipschitz and admits a bounded upper semiregular convexifactor $\partial^{u s} f(\bar{x})$ at $\bar{x}$. Let $g_{i}, i \in I,-h_{i}, h_{i}, i \in I^{\prime},-G_{i}, G_{i}, i \in A \cup B,-H_{i}$, $H_{i}, i \in D \cup B$, admit upper convexifactors $\partial^{u} g_{i}(\bar{x}), i \in I, \partial^{u}\left(-h_{i}\right)(\bar{x}), \partial^{u} h_{i}(\bar{x}), i \in I^{\prime}, \partial^{u}\left(-G_{i}\right)(\bar{x}), \partial^{u} G_{i}(\bar{x})$, $i \in A \cup B, \partial^{u}\left(-H_{i}\right)(\bar{x}), \partial^{u} H_{i}(\bar{x}), i \in D \cup B$, respectively at $\bar{x}$. Suppose that pos $\mathcal{A}$ is closed and that there exists a partition $\left(B_{1}, B_{2}\right)$ of $B$ such that $\partial^{*}-A C Q\left(B_{1}, B_{2}\right)$ holds at $\bar{x}$. Then, $\bar{x}$ is a generalized alternatively stationary point.

Proof. The beginning of the proof of Theorem 4.4 from [8] remains correct. However, from line 6 on page 1624 until the end of the proof, the argument should be corrected as the following.

$$
\sup _{\eta \in \operatorname{conv} \partial^{u s} f(\bar{x})}\langle\eta, v\rangle \geq 0, \text { for all } v \in \mathcal{A}^{-} .
$$

- Since $\mathcal{A} \subseteq$ pos $\mathcal{A}$, we get

$$
\sup _{\eta \in \operatorname{conv}^{u s} f(\bar{x})}\langle\eta, v\rangle \geq 0, \text { for all } v \in(\operatorname{pos} \mathcal{A})^{-} .
$$


- Since $\partial^{u s} f(\bar{x})$ is also a closed set, conv $\partial^{u s} f(\bar{x})$ is a compact set (see [7], Thm. 1.4.3). By Lemma 3.4, we get

$$
0 \in \operatorname{conv} \partial^{u s} f(\bar{x})+\operatorname{cl}(\operatorname{pos} \mathcal{A}) .
$$

- Since pos $\mathcal{A}$ is closed, we obtain

$$
0 \in \operatorname{conv} \partial^{u s} f(\bar{x})+\operatorname{pos} \mathcal{A} .
$$

Then, there exist scalars $\lambda_{i}^{g} \geq 0, i \in I(\bar{x}), \mu_{i}^{h} \geq 0, \lambda_{i}^{h} \geq 0, i \in I^{\prime}, \mu_{i}^{G} \geq 0, i \in A \cup B_{2}, \lambda_{i}^{G} \geq 0, i \in$ $A \cup B, \mu_{i}^{H} \geq 0, i \in D \cup B_{1}$, and $\lambda_{i}^{H} \geq 0, i \in D \cup B$, such that

$$
0 \in\left[\begin{array}{l}
\operatorname{conv} \partial^{u s} f(\bar{x})+\sum_{i \in I(\bar{x})} \lambda_{i}^{g} \operatorname{conv} \partial^{u} g_{i}(\bar{x})+\sum_{i \in I^{\prime}} \mu_{i}^{h} \operatorname{conv} \partial^{u} h_{i}(\bar{x})+\sum_{i \in I^{\prime}} \lambda_{i}^{h} \operatorname{conv} \partial^{u}\left(-h_{i}\right)(\bar{x}) \\
+\sum_{i \in A \cup B_{2}} \mu_{i}^{G} \operatorname{conv} \partial^{u} G_{i}(\bar{x})+\sum_{i \in A \cup B} \lambda_{i}^{G} \operatorname{conv} \partial^{u}\left(-G_{i}\right)(\bar{x}) \\
+\sum_{i \in D \cup B_{1}} \mu_{i}^{H} \operatorname{conv} \partial^{u} H_{i}(\bar{x})+\sum_{i \in D \cup B} \lambda_{i}^{H} \operatorname{conv} \partial^{u}\left(-H_{i}\right)(\bar{x})
\end{array}\right] .
$$

- Setting

$$
\left\{\begin{array}{l}
\mu_{i}^{G}=0, \quad \forall i \in D \cup B_{1} \\
\mu_{i}^{H}=0, \quad \forall i \in A \cup B_{2} \\
\lambda_{i}^{G}=0, \quad \forall i \in D \\
\lambda_{i}^{H}=0, \quad \forall i \in A
\end{array}\right.
$$

we obtain (4.1), (4.2) and (4.3). The proof is then finished.

\section{Conclusions}

In the paper [8], the author investigated a mathematical programs with equilibrium constraints. The main result, Theorem 4.4 of [8], and the lemma ([8], Lem. 2.3) on which the author is based are false. In this work, counterexamples are given to refute Theorem 4.4 of [8] and Lemma 2.3 of [8]. Furthermore, we correct the flaws.

Acknowledgements. Our sincere acknowledgements to the anonymous referees for their insightful remarks and suggestions.

\section{REFERENCES}

[1] A.A. Ardali, N. Movahedian and S. Nobakhtian, Optimality conditions for nonsmooth mathematical programs with equilibrium constraints, using convexifactors. Optimization 65 (2016) 67-85.

[2] J. Dutta and S. Chandra, Convexifactors, generalized convexity, and optimality conditions. J. Optim. Theory App. 113 (2002) $41-64$.

[3] M.L. Flegel, Constraint qualifications and stationarity concepts for mathematical programs with equilibrium constraints. Doctoral dissertation, Universität Würzburg (2005).

[4] M.L. Flegel and C. Kanzow, A Fritz John approach to first-order optimality conditions for mathematical programs with equilibrium constraints. Optimization 52 (2003) 277-286.

[5] M.L. Flegel and C. Kanzow, Abadie-Type constraint qualification for mathematical programs with equilibrium constraints. J. Optim. Theory App. 124 (2005) 595-614.

[6] M.L. Flegel and C. Kanzow, On the guignard constraint qualification for mathematical programs with equilibrium constraints. Optimization 54 (2005) 517-534.

[7] J.B. Hiriart-Urruty and C. Lemarechal, Fundamentals of Convex Analysis. Springer-Verlag, Berlin Heidelberg (2001).

[8] B. Kohli, Necessary and sufficient optimality conditions using convexifactors for mathematical programs with equilibrium constraints. RAIRO:OR $\mathbf{5 3}$ (2019) 1617-1632. 
[9] H. Scheel and S. Scholtes, Mathematical programs with complementarity constraints: stationarity, optimality, and sensitivity. Math. Oper. Res. 25 (2000) 1-22.

[10] J.J. Ye, Necessary and sufficient optimality conditions for mathematical programs with equilibrium constraints. J. Math. Anal. App. 307 (2005) 350-369.

\section{Subscribe to Open (S20) A fair and sustainable open access model}

This journal is currently published in open access under a Subscribe-to-Open model (S2O). S2O is a transformative model that aims to move subscription journals to open access. Open access is the free, immediate, online availability of research articles combined with the rights to use these articles fully in the digital environment. We are thankful to our subscribers and sponsors for making it possible to publish this journal in open access, free of charge for authors.

\section{Please help to maintain this journal in open access!}

Check that your library subscribes to the journal, or make a personal donation to the S2O programme, by contacting subscribers@edpsciences.org

More information, including a list of sponsors and a financial transparency report, available at: https://www. edpsciences.org/en/maths-s2o-programme 